\title{
Assessment of precision in ionospheric electron density profiles retrieved by GPS radio occultations
}

\author{
P. Alexander ${ }^{1, *}$ \\ IFIBA, CONICET, Ciudad Universitaria Pabellón 1, 1428 Buenos Aires, Argentina \\ A. de la Torre, R. Hierro and P. Llamedo ${ }^{2, *}$ \\ Facultad de Ingeniería, Universidad Austral, Av. J. de Garay 125, 1063 Buenos Aires, \\ Argentina
}

\begin{abstract}
The Constellation Observing System for Meteorology Ionosphere and Climate (COSMIC) is a six satellite radio occultation mission that was launched in April 2006. The close proximity of these satellites during some months after launch provides a unique opportunity to evaluate the precision of Global Positioning System (GPS) radio occultation (RO) retrievals of ionospheric electron density from nearly collocated and simultaneous observations. RO data from 30 consecutive days during July and August 2006 are divided into ten groups in terms of daytime or nighttime and latitude. In all cases, the best precision values (about 1\%) are found at the F peak height and they slightly degrade upwards. For all daytime groups, it is seen that electron density profiles above about $120 \mathrm{~km}$ height exhibit a substantial improvement in precision. Nighttime groups are rather diverse: in particular, the precision becomes better than $10 \%$ above different levels between 120 and $200 \mathrm{~km}$ height. Our overall results show that up to 100-200 km (depending on each group), the uncertainty associated with the precision is in the order of the measured electron density values. Even worse, the retrieved values tend sometimes to be negative. Although we cannot rely directly on electron density values at these altitudes, the shape of the profiles could be indicative
\end{abstract}

\footnotetext{
${ }^{*}$ Corresponding author

Email address: peter@df.uba.ar (P. Alexander)

${ }^{1}$ Telephone 54-11-4576-3353, Fax 54-11-4576-3357

2adelaTorre@austral.edu.ar,rhierro@austral.edu.ar,pllamedo@austral.edu.ar
} 
of some ionospheric features (e.g. waves and sporadic E layers). Above $200 \mathrm{~km}$, the profiles of precision are qualitatively quite independent from daytime or latitude. From all the nearly collocated pairs studied, only 49 exhibited a difference between line of sight angles of both RO at the F peak height larger than $10^{\circ}$. After analyzing them we find no clear indications of a significant representativeness error in electron density profiles due to the spherical assumption above $120 \mathrm{~km}$ height. Differences in precision between setting and rising GPS RO may be attributed to the modification of the processing algorithms applied to rising cases during the initial period of the COSMIC mission.

Keywords: GPS radio occultation, electron density, uncertainties

\section{Introduction}

A Global Positioning System (GPS) radio occultation (RO) occurs whenever a transmitting satellite at an altitude about 20,000 km rises or sets from the standpoint of a low Earth orbit (LEO) satellite orbiting at a height of about $500 \mathrm{~km}$. The aim of the GPS RO method is to detect the Doppler frequency perturbation produced by the refraction of the signal in the Earth's atmosphere along the path between the transmitter and the receiver. This information can be then converted into profiles of variables in the neutral atmosphere and ionosphere (Pavelyev et al., 2007, 2010). The measurement time of the technique in the ionosphere is typically $4 \mathrm{~min}$ (as compared to much longer ionospheric processes), it has global coverage and exhibits a good vertical resolution (about $2 \mathrm{~km}$ ).

The Constellation Observing System for Meteorology, Ionosphere and Climate (COSMIC) was launched in April 2006, with the aim of producing about 2500 GPS RO daily across the globe. The orbital characteristics of the six LEO satellites imply that most $\mathrm{RO}$ retrievals occur at mid-latitudes. Immediately after launch, the six LEO satellites orbited in very close proximity for a few months, so during this period the data was particularly clustered (e.g., Liou et al., 2007). This offers an excellent opportunity to evaluate the precision of the retrieved electron density through the use of nearly collocated and simultaneous observations with respect to one given GPS satellite, whereby the RO planes are almost parallel. The corresponding retrievals therefore include almost the same sampled region and time.

Measurement errors arise in processes where the instruments interact ei- 
ther directly or indirectly with the quantity being measured. In the latter case, representativeness errors emerge quite naturally, their relevance depending on how well the designed methodology represents and truly measures the targeted variable. For example, measurements in the atmosphere are often based on the mathematical processing of remote-sensing observations, which includes implicit or explicit underlying assumptions. Measurements obtained from different LEO satellites at the same time may be assigned to the same spatial coordinates, but they may be weighted averages along different lines of sight (LOS) along that point (to make it simple, one could be along the North-South direction, the other one along the West-East direction). This can lead to significantly different values for the same coordinates in space and time, unless the spherical symmetry assumption holds around the point, which is usually not true. In fact, that is the main cause of error in the GPS $\mathrm{RO}$ electron density profile retrievals because the ionosphere is typically not horizontally homogeneous (e.g., Schreiner et al., 1999; Wu et al., 2009).

In general, differences in measurements assigned to a point and time that are obtained from diverse GPS RO depend on two factors: the precision of the observations and the representativeness errors that arise because different regions of the atmosphere are being sounded (Staten and Reichler, 2009). In order to isolate the contribution of precision to those measurement differences, the existence of pairs of GPS RO that are nearly simultaneous and collocated becomes quite relevant (two different soundings are assigning measurements to almost the same points of space at almost the same times). Precision here refers to the level of "repeatability" of GPS RO measurements under the same conditions. Nearly collocated pairs of GPS RO ensure that the sounded areas for each measurement are nearly the same, as both LEO satellites are very close and face the same complementary GPS satellite. This keeps measurement differences stemming from representativeness errors to a minimum, but of course does not eliminate them. However, the nearly collocated retrievals are the best possibility we presently have to separate precision issues from representativeness errors. There is another feature that is related to representativeness errors, which we cannot address here because it depends on the specific use of the RO data in each case: a possible scale mismatch between the sounded region for each measurement and the intended application of the observations. GPS RO ionospheric data typically represent horizontal averages over $1000 \mathrm{~km}$, so an uncertainty clearly emerges if a study requires, for example, a knowledge of the electron density representing at each point a horizontal scale of about $10 \mathrm{~km}$. 
The precision of an instrument or an observational technique may be assessed by the root-mean-square (RMS) difference between a large number of pairs of independent observations. In the case of GPS RO retrievals of ionospheric data we apply this procedure to evaluate the electron density at altitudes within the observable range. It must be noted that measurements usually cannot be repeated under exactly the same conditions. Here, we try to keep the separations in time and space of pairs of retrievals as small as possible. The fact that in the early stages of COSMIC the LEO satellites were close to each other helped to achieve this objective. Thus, the precision investigated below is mainly due to the observational error between different receivers and may also include the numerical error of the processing software. GPS RO error inter-comparison studies for different missions focused mostly on precision and representativeness issues in the neutral atmosphere (e.g., Hajj et al., 2004; Schreiner et al., 2007; Staten and Reichler, 2009; Alexander et al., 2010). For this reason, such diversity of studies is presently lacking for ionospheric retrievals (Schreiner et al., 2007).

Accuracy (level of "exactitude") of the GPS RO retrieval method has been theoretically evaluated (e.g., Kursinski et al., 1997) and experimentally assessed by contrast with observational data from other sources (Hajj and Romans, 1998; Jakowski et al., 2002). However, it should be taken into account that any such comparison encompasses not only the measurement but also the representativeness errors of any two observational methods being compared. Representativeness errors for radiosonde observations have been evaluated by Kitchen (1989). The GPS RO electron density accuracy is mainly related to the spherical symmetry assumption within the retrieval. As explained above, the use of nearly collocated pairs allowed to separate precision from accuracy issues. A problem in the evaluation of GPS RO accuracy is that other platforms do not have its global and permanent characteristic, so inter-comparisons can only be established in some space and time isolated cases. Then, accuracy cannot in general be analyzed at the same level of detail as precision, as there are no simultaneously collocated global and reliable reference measurements available.

Section 2 shows the GPS RO retrieval method used to obtain electron density profiles in the ionosphere by CDAAC (COSMIC Data Analysis and Archive Center). In section 3 we describe the corresponding database used in this study, the selection of processable pairs of retrievals through a proximity criterion, their classification in groups in terms of daytime and latitude and the analysis methodology. In section 4 we present the results. Section 5 con- 
tains a discussion of the possible relevance of the results. Finally, conclusions are drawn.

\section{The GPS RO Electron Density Retrieval Technique}

When a signal is transmitted from a GPS to a LEO satellite and it passes through the Earth's atmosphere in a limb sounding geometry, its phase and amplitude are affected according to the index of refraction of the medium along the path. Each RO observation consists of a set of limb-viewing links with measurement points ranging from the LEO satellite orbit altitude to the surface of the Earth. Due to the dispersive propagation properties of the ionosphere for radio signals, the limb-viewing total electron content $(T E C)$ values can be derived from received signal measurements. Signal phase delay from LEO to GPS satellites is related basically to the limb-viewing TEC in the ionosphere. Observed phase changes between the source and the signal detection may ultimately lead to electron density profiles if certain assumptions are made (mainly spherical symmetry around the measurements). The processing technique for extracting ionospheric delay makes use of two signals to obtain $T E C$, which is derived from a linear combination of such signals. The assumption of spherical symmetry is not optimal regarding electron density measurement accuracy, but as it often happens in science, there is no better choice for the moment, so it remains the most robust source of global electron density profiles. At the same time, it forces us to consider the results of any analysis of retrievals in an adequate context. For example, we must keep in mind that the results have very low horizontal resolutions, in the order of $1000 \mathrm{~km}$ or even more (Hajj et al., 2000) as shown in Figure 1a. The vertical resolution of an ionospheric RO depends on the receiver sampling interval and the measurement point ascent (descent) rate, but is essentially limited to about $1.5 \mathrm{~km}$.

GPS RO observations are assigned to the tangent point to the Earth of every straight line ray (bending is negligible) traveling from the transmitting to the receiving satellite. The distance from the center of the Earth to a point on a ray between both satellites is $r$ and to the corresponding tangent point it is $r_{o}$, whereas the distance along the ray starting from the tangent point is $l$. The description below outlines the essential scheme currently used at CDAAC to derive electron density profiles from the COSMIC GPS RO observations. If phase paths of two different signals $L_{1}$ and $L_{2}$ are expressed 
in distance units (phase times the light speed divided by the frequency) (Schreiner et al., 1999)

$$
T E C=\int_{L E O}^{G P S} N_{e} d l=\frac{\left(L_{1}-L_{2}\right){f_{1}}^{2}{f_{2}}^{2}}{40.3\left({f_{1}}^{2}-{f_{2}}^{2}\right)}
$$

where $N_{e}$ is electron density and the signal frequencies are $f_{1}=1.57542 \mathrm{GHz}$ and $f_{2}=1.22760 \mathrm{GHz}$. The derived $T E C$ is then calibrated such that it represents the portion below the LEO orbit (Schreiner et al., 1999), which is done by subtracting auxiliary TEC measured at the opposite position of the LEO orbit (see Figure 1b). If the auxiliary data are not available due to the relative motion of the satellites, then only the information at the LEO altitude will be accessible and the missing auxiliary $T E C$ at lower tangent point heights becomes modeled through the additional fit of a functional expression for $N_{e}$ above the LEO orbit. Figure 1a implies that $r^{2}=l^{2}+r_{o}^{2}$, so under the assumptions of spherical symmetry (there are only vertical electron density gradients) and straight-line ray propagation it follows from Eq. (1) that

$$
T E C\left(r_{o}\right)=\int_{r_{o}}^{r_{G P S}} \frac{r N_{e}(r)}{\sqrt{r^{2}-r_{o}^{2}}} d r+\int_{r_{o}}^{r_{L E O}} \frac{r N_{e}(r)}{\sqrt{r^{2}-r_{o}^{2}}} d r
$$

whereas the calibrated $T E C^{\prime}$ due to the spherical symmetry is

$$
T E C^{\prime}\left(r_{o}\right) \approx 2 \int_{r_{o}}^{r_{L E O}} \frac{r N_{e}(r)}{\sqrt{r^{2}-r_{o}^{2}}} d r
$$

For Eq. (3) it is also assumed that the GPS satellite is contained in or close to the LEO orbit plane and that the ionospheric conditions of the sounded region stay nearly constant during the period of measurement. The equation can be inverted (see Appendix A) by using an Abel integral transform (e.g., Tricomi, 1985)

$$
N_{e}(r)=-\frac{1}{\pi} \int_{r}^{r_{L E O}} \frac{d T E C^{\prime}\left(r_{o}\right) / d r_{o}}{\sqrt{r_{o}^{2}-r^{2}}} d r_{o}
$$

Although the obtained $N_{e}$ is limited by the accuracy of the measured GPS signal phases, non-spherical symmetric real electron density is thought to be the main source of error. The problem becomes magnified when the ray propagates through the lower ionospheric layers. 
The numerical integration of Eq. (4) may lead to difficulties due to a singular behavior of the integrand close to the top height. An alternative way to overcome this difficulty is to split the right-hand side of Eq. (3) into the sum of $m$ integrals in consecutive shells. Assuming that $N_{e}$ varies linearly with the distance from the center of the Earth in between each shell, the $m$ integrals can be solved analytically and $T E C^{\prime}$ at any level can be obtained in terms of the electron density at this and the upper $m$ levels. This triangular linear system of equations can then be inverted to yield $N_{e}$ at any level $i$ in terms of $T E C^{\prime}$ at that level and $N_{e}$ at $m$ levels above (e.g., Lei et al., 2007):

$$
N_{e}\left(r_{o i}\right)=\frac{1}{c_{i, 0}}\left(\frac{T E C^{\prime}\left(r_{o i}\right)}{r_{o i}}-\sum_{k=1}^{m} c_{i, k} N_{e}\left(r_{o(i+k)}\right)\right)
$$

where $c_{i, 0}$ and $c_{i, k}$ are very long dimensionless expressions that depend on the relative location of a level between the tangent point and the LEO orbit. Now we can calculate the $N_{e}$ profile recursively starting from the top (the LEO orbit) if we know its value there. An estimate can be obtained by assuming that $N_{e}$ close to the top is a constant. Under this assumption, the integral in Eq. (3) can be solved to give

$$
T E C^{\prime}\left(r_{o}\right)=2 N_{e}\left(r_{L E O}\right) \sqrt{2 r_{L E O}\left(r_{L E O}-r_{o}\right)}
$$

\section{Data and Processing}

This study uses the latest post-processed data (version 2010.2640) from the COSMIC mission provided by CDAAC. We statistically evaluate the precision of GPS RO in the ionosphere by using the COSMIC level 2 electron density product, which is the most valuable quantity measured in this atmospheric region using this technique. We used 69,016 GPS RO available from days 194-223 during July and August 2006. We used only 30 days in our study, in order to minimize any seasonal data variation. There is a delicate balance between narrowing the acceptable separation standard in space and time of both soundings of every pair (to ensure that we are measuring the "same" observable), and keeping a large number of cases so as to be able to perform satisfactory statistics. We chose to examine all pairs with a time and horizontal separation of respectively less than 1 min between both soundings and less than $10 \mathrm{~km}$ between the corresponding tangent points at every altitude. This criterion yielded 2892 pairs and thus allowed us to 
obtain good statistical power. A similar analysis was performed by Schreiner et al. (2007). Although they applied a stricter criterion (5 km separation) because the retrievals they used were nearer to the launching date, their data was produced with the initial processing algorithms of COSMIC. Also, our study separates the data in terms of daytime or nighttime and latitude zones.

We classified the pairs into 10 groups. Their names are DHN, DHS, DMN, DMS, DL, NHN, NHS, NMN, NMS and NL, where the first letter refers to daytime $(\mathrm{D})$ or nighttime $(\mathrm{N})$ and the second and/or third letter refer to low $(\mathrm{L})$, middle $(\mathrm{M})$ and high $(\mathrm{H})$ latitudes in the Northern $(\mathrm{N})$ and Southern (S) Hemispheres. The geographic latitudes that define the zones correspond to $\pm 20^{\circ}$ and $\pm 55^{\circ}\left(180^{\circ} \mathrm{W}-180^{\circ} \mathrm{E}\right)$. Events within \pm 30 minutes of sunrise or sunset were excluded (about $10 \%$ ) in order to construct groups as homogeneous as possible. In Table 1 we show the number of pairs per group.

Profiles were analyzed between $60 \mathrm{~km}$ and $530 \mathrm{~km}$ altitudes and we did not exclude cases with unrealistic negative electron density values. Most retrieved profiles reached a maximum height somewhere between 500 and $530 \mathrm{~km}$. Those early COSMIC surveys did not reach top altitudes above $800 \mathrm{~km}$ due to their working orbits at that time. All the pairs detected belong to satellites 3 and 4, so we were not able to find triads of close RO. In Figure 2 we show the number of RO retrievals per day for satellite 4 (as a proxy for the potential number of nearly collocated and simultaneous cases) and the number of pairs found. These two quantities show a decreasing trend over time when analyzing several months. It is therefore expected that the statistical significance of additional months of data will decline, and in addition it could also reduce the data homogeneity degree, as seasonal effects could start to transfer variability into the results.

We first calculated the average electron density profile for each of the ten groups. In order to check the homogeneity degree of the group we also computed the standard deviation of the profiles. This calculation helps to see whether the classification in groups separates the profiles in terms of similarity, at least for some height ranges, or if there is a large dispersion among the characteristics of the curves in each of the ten sets. Thereafter, we calculated the RMS of the differences in measured electron density of all pairs in each group against height. The standard deviation and RMS values were normalized in each group to the mean electron density in order to have relative rather than absolute proxies, which may be more adequate measures of the homogeneity and precision degrees. This is particularly applicable to 
the present analysis because: i) the calculated standard deviation and RMS can change by orders of magnitude with height because the electron density does so, ii) some values of the standard deviation and RMS may be tricky, as they may apparently look small, but at the lowest heights they may be very significant as the electron density stays about zero (the ratios give us a warning when they become very large).

To analyze the consequences of the spherical symmetry assumption, which may be considered associated to the representativeness error, we separately consider a few COSMIC pairs which exhibit significantly different LOS angles in both retrieved profiles. Although the location of the two profiles is nearly the same, they might be sampling largely different ionospheric features along the different path integrals. We selected those pairs which had an angle difference larger than $10^{\circ}$ at the height of the $\mathrm{F}$ region electron density peak. More than $90 \%$ of the pairs have a difference lower than $1^{\circ}$. We computed the RMS for two sets defined by angle differences within the $10-20^{\circ}$ and $20-40^{\circ}$ ranges, which respectively had 21 and 28 pairs. We compared both results among them and with the overall values (that include a vast majority of very small angle differences). However, we should mention that this kind of study may not be strong enough to demonstrate the effects of the spherical symmetry assumption.

We also assessed any possible statistical differences between rising and setting events, which may contain precision issues. A priori there should be no differences in the outcome, but this problem should not be directly excluded. Rising and setting RO needed modifications in processing algorithms over time (Schreiner et al., 2007). We compared the RMS against height for both types in one of the ten groups in order to see if they produce a significantly different outcome.

The precision results below correspond to a 30 day (194-223) of year 2006 data study. In order to verify if there is statistical robustness in the results, we first performed all the calculations for the initial 15 days (194-208). We compared the statistical outcomes of both sets and found that doubling the amount of days (and roughly also the amount of available close RO pairs) did not lead to qualitative differences, only to small quantitative variations (we therefore do not show the results for the smaller data-set). Even the degree of homogeneity of the data was not significantly affected (one could expect seasonal variations to have some impact with increasing time intervals). We may then consider that the results below in terms of 8 characteristic groups are statistically representative of the GPS RO measurement properties. The 
amounts of GPS RO pairs for DHS and NHN are not significant, so both groups have been removed from any further discussion.

\section{Results}

Some results among the various groups are quite similar, so for brevity we now exhibit figures only for three representative cases. The daytime mean electron density profile for group DL is shown in panel a of Figure 3, whereas the same is displayed for the nighttime groups NHS and NMN in Figures 4 and 5 . In addition, in panels $\mathrm{b}$ we show the number of pairs to evaluate the statistical strength in each case, in panels c we exhibit the ratio of the standard deviation and the mean of the electron density to assess the homogeneity of each group and in panels $d$ we plot the ratio of the RMS of the pairs and the mean electron density of each group. The last quantity addresses the issue of the precision of the profiles of a given group in terms of altitude. However, it should not be considered separately from the other panels to evaluate its relevance. Daytime electron density profiles exhibit an $\mathrm{F}$ peak maximum at about $200-270 \mathrm{~km}$ height, with increasing altitude towards the Equator and larger values in the summer hemisphere. The $\mathrm{F}$ region usually becomes divided during daytime into the lower F1 and the upper F2 parts. In our daytime electron density profiles the minor peak of the F1 layer becomes insinuated at low latitudes and in the Northern Hemisphere (see Figure 3). Nighttime values are generally less intense and F peaks are located at around $250-300 \mathrm{~km}$ and become less intense from the northern mid-latitudes to the South. DL and NMN exhibit unrealistic continuous or discontinuous negative values in the lowest altitudes. Negative values of the ratios in panels $\mathrm{c}$ and $\mathrm{d}$ in Figures 3-5 are excluded by restricting the graphs to the 0-1 range. In all groups, precision is best at the $\mathrm{F}$ peak height and slightly degrades upwards. All daytime mean electron density profiles become more homogeneous above about $120 \mathrm{~km}$ height, which also coincides with a substantial improvement in precision. The lack of homogeneity can be due to the true profiles characteristics or more likely to the difficulty of the GPS RO technique in adequately reproducing the electron density at low heights. Nighttime average profiles have less common features than their daytime counterparts. In NHS, the average electron density profile exhibits a very distinct aspect with respect to all other groups with an almost unnoticeable F peak (night is present in a vast majority of the time in this latitudinal region during winter). Above $120 \mathrm{~km}$ altitude, precision becomes 
good notwithstanding the small electron density values. Both NMN ratios have remarkable spikes around $200 \mathrm{~km}$ height. This is due to the nearly null values that mark the transition of electron density profiles from negative below to positive values above. The unrealistic behavior at low altitudes exists notwithstanding the good statistical power. Some nighttime mean profiles reach good levels of precision from about $200 \mathrm{~km}$. No clear most homogeneous nighttime group can be distinguished.

From the 2602 GPS RO pairs analyzed, there were just 49 that exhibited a difference larger than $10^{\circ}$ between both LOS angles at the $\mathrm{F}$ peak height. The general common factor among the 49 cases is that they are grazing RO. This means that although they have been finally classified as rising or setting, they lie very close to the boundary of both possibilities, so they show a significant horizontal displacement of the tangent points with height (up to almost $1000 \mathrm{~km}$ ). The relevance of this kind of geometry and obliquity of profiles has been assessed in the neutral atmosphere by Foelsche et al. (2011). Figure 6 a shows the ratio of RMS and mean electron density against height for the 21 pairs which have an angle difference between $10^{\circ}$ and $20^{\circ}$, whereas Figure $6 \mathrm{~b}$ shows the same for the 28 cases within the interval between $20^{\circ}$ and $40^{\circ}$. There is no visible degradation in the second case with respect to the first one. The statistical power is too low to infer that the spherical assumption is apparently not as costly as presently assumed. Both sets perform very similarly. However, this may be a consequence of the particular characteristics of the technique, where the value assigned to every tangent point is some kind of electron density average over large distances. The reduced quality at the lowest heights could be attributed to the spherical symmetry assumption, but may also be due to general difficulties of the RO method in describing the features at those heights. When comparing Figure 6 with panels d of Figures 3-5 one sees no significant differences in precision.

We also analyzed any possible issues between setting and rising GPS RO. We focused on the DMN group, which is among the most homogeneous ones and has good statistical power. We can see that up to about $120 \mathrm{~km}$ altitude neither type performs well, but from there upwards they both do much better. However, there is clearly a better precision in the setting RO in Figure 7a as compared to rising $\mathrm{RO}$ in $7 \mathrm{~b}$.

We made our own assessment of the relevance of the negative electron densities in general with a GPS RO data-set different from the one used above for the assessment of precision. These new data belong to a much longer period, as we were not compelled to use nearly collocated pairs for 
this evaluation. We analyzed 1 full day of COSMIC profiles for every month of year 2011. Sunrise and sunset cases have been discarded as above in our study. We searched for anomalous negative cases between 100 and 200 $\mathrm{km}$ height (the typical range where this occurs). An amount of 10,822 RO have been studied, whereby $46.4 \%$ of nighttime and $20.8 \%$ of daytime RO were negative in at least one point. Measured either by percentage of cases or negative intensity, the most significant effect is found at low latitudes. There was no significant difference between rising and setting RO (33\% were anomalous in both cases). There was also no significant difference in terms of LOS angle. In both hemispheres maximum/minimum effect is found during winter/summer.

\section{Discussion}

We recall some issues regarding the unrealistic negative electron density. A document describing the algorithms used at CDAAC states that the inversion of RO signals in the ionosphere is based on the assumption of local spherical symmetry of the electron density in a large region (a few thousand kilometers in radius) around the ray path tangent points, but that this assumption may not always be valid, so horizontal ionospheric gradients may significantly affect the retrieved electron density profiles, in particular below the $\mathrm{F}$ layer, sometimes giving large negative or positive values (cdaacwww.cosmic.ucar.edu/cdaac/doc/documents/gmrion.pdf). A much earlier report (Høeg et al., 1998) had already warned that when the assumption of spherical symmetry is violated, the calculated electron density can become negative. Worst asymmetric cases for $\mathrm{RO}$ were simulated in a daytime to nighttime transition region, resulting in large positive or negative biases, particularly at the lowest heights. In addition, it was stated that a side-viewing RO would normally give a higher error in the profiles than a straight-on perspective, because the former is scanned over a much larger path. It is therefore more likely that the assumption of spherical symmetry has been violated.

The derivation of electron density inevitably becomes significantly affected at the lowest tangent points (mainly at $\mathrm{D}$ and E layers) by the stronger effect on the occultation ray traversing the ionosphere at higher altitudes (recall Figure 1). In fact, the best precision results in this work are found around the electron density $\mathrm{F}$ peak (about 1\%) and they deteriorate both upwards and downwards. The result is in agreement with Schreiner et al. 
(2007). In particular, these authors had already seen large RMS around 100 $\mathrm{km}$ altitude and they considered it likely that these results derived from a possible combination of effects: the inability of GPS RO to perform a good sampling of sharp vertical structures, the effect of horizontal variations due to sporadic events in the E-layer, signal defocusing due to large gradients, natural spatial variations and, to a lesser extent, thermal noise. In the lowest altitudes but well above about $100 \mathrm{~km}$, the worse precision is mainly due to the accumulated error from the $\mathrm{F}$ layer and below. In the highest altitudes the problem is mainly due to an upper boundary calibration issue (Yue et al., 2011). In all daytime groups precision is better than $10 \%$ above $120 \mathrm{~km}$ altitude. For the nighttime cases, precision generally improves above about $200 \mathrm{~km}$ height and from there upwards the best performance is found in the Northern Hemisphere (summer during the studied period). Moreover, NHS is the only group that exceeds a $10 \%$ precision about $500 \mathrm{~km}$ height. In our overall results we have seen that up to 100-200 km (depending on each group), the uncertainty associated with the precision is in the order of the measured electron density values, or the outcome is even worse: the retrieved values may be negative, whereby the most remarkable group in our study is NMN. We must conclude that we cannot be too confident about the electron density values at these altitudes in general, but some reliability can perhaps be assigned to the shape of the profiles (e.g. waves and sporadic E layers).

\section{Conclusions}

We take advantage of the fact that immediately after launch the data of the COSMIC mission from different satellites were clustered to evaluate the precision of the electron densities retrieved by GPS RO. We divided the data into groups according to latitude bands and daytime or nighttime. In all cases, the best standards are found at the $\mathrm{F}$ peak height (about 1\%), which slightly degrade upwards (only in NHS it exceeds $10 \%$ around $500 \mathrm{~km}$ height). In general, the precision becomes better than $10 \%$ above about 120 $\mathrm{km}$ height for the daytime groups. The same occurs for the nighttime groups above about $200 \mathrm{~km}$ altitude. In our overall results we have seen that up to 100-200 km (depending on each group), the uncertainty associated with the precision is in the order of the measured electron density values. Even worse, the retrieved values may be sometimes negative. We cannot rely on electron density values at these altitudes in general, but if we speculate that there is a nearly constant negative bias, the shape of the profiles could be indicative 
of some ionospheric features (e.g. waves and sporadic E layers). We may infer that nighttime groups are in general less homogeneous and precise because they tend to have electron density values closer to zero (mainly at the lowest altitudes), so they are more affected by variabilities and uncertainties. Notwithstanding daytime or latitude variation, above $200 \mathrm{~km}$ the profiles of precision are qualitatively quite similar. From all the pairs analyzed, there were only 49 that exhibited a difference between LOS angles of both RO at the $\mathrm{F}$ peak height larger than $10^{\circ}$. From the analysis of the 49 cases, we find no clear representativeness error introduced by the spherical assumption above $120 \mathrm{~km}$ height. However, these results have a low statistical power. The differences in precision found here between setting and rising GPS RO may be attributed to the fact that processing algorithms were modified during the initial period of the COSMIC mission.

Acknowledgements Manuscript prepared under grant CONICET PIP 11220090100649. P. Alexander, A. de la Torre, R. Hierro and P. Llamedo are members of CONICET. GPS RO data downloaded from cdaac-ftp.cosmic.ucar.edu.

Alexander, P., Luna, D., de la Torre, A., Llamedo, P., Schmidt, T., Wickert, J. (2010), A comparative and numerical study of effects of gravity waves in small miss-distance and miss-time GPS radio occultation temperature profiles, Adv. Space Res., 45, 1231-1234.

Foelsche, U., Syndergaard, S., Fritzer, J., Kirchengast, G. (2011), Errors in GNSS radio occultation data: relevance of the measurement geometry and obliquity of profiles, Atmos. Meas. Tech., 4, 189-199.

Hajj, G. A. and Romans, L. J.: Ionospheric electron density profiles obtained with the Global Positioning System: Results from the GPS/MET experiment (1998), Radio Sci., 33, 175-190.

Hajj, G. A., Lee, L. C., Pi, X., Romans, L. J., Schreiner, W. S., Straus, P. R., Wang, C. (2000), COSMIC GPS ionospheric sensing and space weather, Terr. Atmos. Oceanic. Sci., 11, 235-272.

Hajj, G. A., Ao, C. O., Iijima, B. A., Kuang, D., Kursinski, E. R., Mannucci, A. J., Meehan, T. K., Romans, L. J., Juarez, M. D., Yunck, T. P. (2004), CHAMP and SAC-C atmospheric occultation results and intercomparisons, J. Geophys. Res., 109, D06109. 
Høeg, P., Larsen, G. B., Benzon, H.-H., Grove-Rasmussen, J., Syndergaard, S., Mortensen, M. D., Christensen, J., Schultz, K. (1998), GPS atmosphere profiling methods and error assessments, Scientific Report 98-7, Section 4.1.4, Danish Meteorological Institute, Copenhagen, Denmark.

Jakowski, N., Wehrenpfennig, A., Heise, S., Reigber, Ch., Lhr, H., Grunwaldt, L., Meehan, T.K. (2002), GPS radio occultation measurements of the ionosphere from CHAMP: Early results, Geophys. Res. Lett., 29, 1457.

Kitchen, M. (1989), Representativeness errors for radiosonde observations, Q. J. R. Meteorol. Soc., 115, 673-700.

Kursinski, E. R., Hajj, G. A., Schofield, J. T., Linfield, R. P., Hardy, K. R. (1997), Observing Earth's atmosphere with radio occultation measurements using the Global Positioning System, J. Geophys. Res., 102, 42923,465 .

Lei, J., et al. (2007), Comparison of COSMIC ionospheric measurements with ground-based observations and model predictions: Preliminary results, $J$. Geophys. Res., 112, A07308, doi:10.1029/2006JA012240.

Liou, Y.-A., Pavelyev, A. G., Liu, S.-F., Pavelyev, A. A., Yen, N., Huang, C.-Y., Fong, C. J. (2007), FORMOSAT-3/COSMIC GPS radio occultation mission: preliminary results, IEEE Trans. Geosci. Remote Sens., 45, 38133826 .

Pavelyev, A. G., Liou, Y. A., Wickert, J., Schmidt, T., Pavelyev, A. A. and Liu, S. F. (2007), Effects of the ionosphere and solar activity on radio occultation signals: Application to CHAllenging Minisatellite Payload satellite observations, J. Geophys. Res., 112, A06326, doi:10.1029/2006JA011625.

Pavelyev, A.G., Liou, Y.-A., Wickert, J., Zhang, K., Wang, C.-S. and Kuleshov, Y. (2010), Analytical model of electromagnetic waves propagation and location of inclined plasma layers using occultation data, Progress in Electromagnetics Research (PIER), 177-202, doi: 10.2528/PIER10042707.

Staten, P. W. and Reichler, T. (2009), Apparent precision of GPS radio occultation temperatures, Geophys. Res. Lett., 36, L24806. 
Schreiner, W. S., Sokolovskiy, S. V., Rocken, C., Hunt, D. C. (1999), Analysis and validation of GPS/MET radio occultation data in the ionosphere, Radio Sci., 34, 949-966.

Schreiner, W. S., Rocken, C., Sokolovskiy, S.,Syndergaard, S., Hunt, D. (2007), Estimates of the precision of GPS radio occultations from the COSMIC/FORMOSAT-3 mission, Geophys. Res. Lett., 34, L04808.

Tricomi, F. G. (1985), Integral Equations, pp. 238, Dover, Mineola, N. Y.

Wu, X., Hu, X., Gong, X., Zhang, X., and Wang, X. (2009), Analysis of inversion errors of ionospheric radio occultations, GPS Solut., 13, 231-239.

Yue, X., W. S. Schreiner, C. Rocken, and Y.]H. Kuo (2011), Evaluation of the orbit altitude electron density estimation and its effect on the Abel inversion from radio occultation measurements, Radio Sci., 46, RS1013. 
Table 1: Number of pairs of close RO in the ten groups.

\begin{tabular}{|c|c|c|c|}
\hline & Daytime & Nighttime & Total \\
\hline $55^{\circ}$ to $90^{\circ}$ & 182 & 3 & 185 \\
$20^{\circ}$ to $55^{\circ}$ & 751 & 154 & 905 \\
$-20^{\circ}$ to $20^{\circ}$ & 220 & 179 & 399 \\
$-55^{\circ}$ to $-20^{\circ}$ & 199 & 746 & 945 \\
$-90^{\circ}$ to $-55^{\circ}$ & 4 & 164 & 168 \\
\hline Total & 1356 & 1246 & 2602 \\
\hline
\end{tabular}




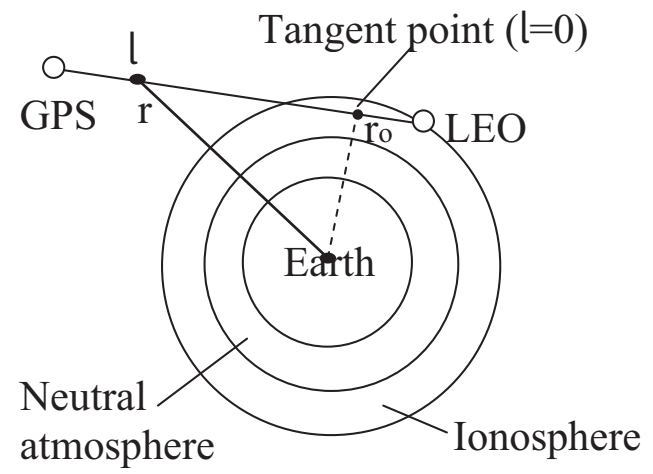

a

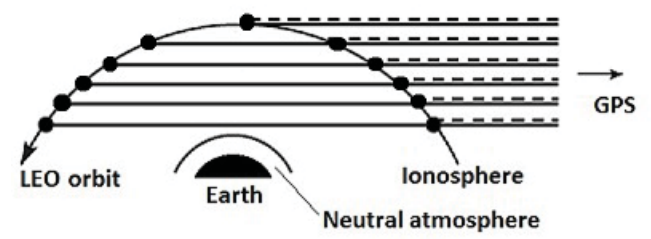

$\mathrm{b}$

18

Figure 1: a) Representation out of scale of the geometry of the GPS-LEO radio occultation for observations in the ionosphere. b) Basic scheme of the calibrated $T E C$ retrieval (solid line $T E C$ minus dotted line $T E C$ ). 


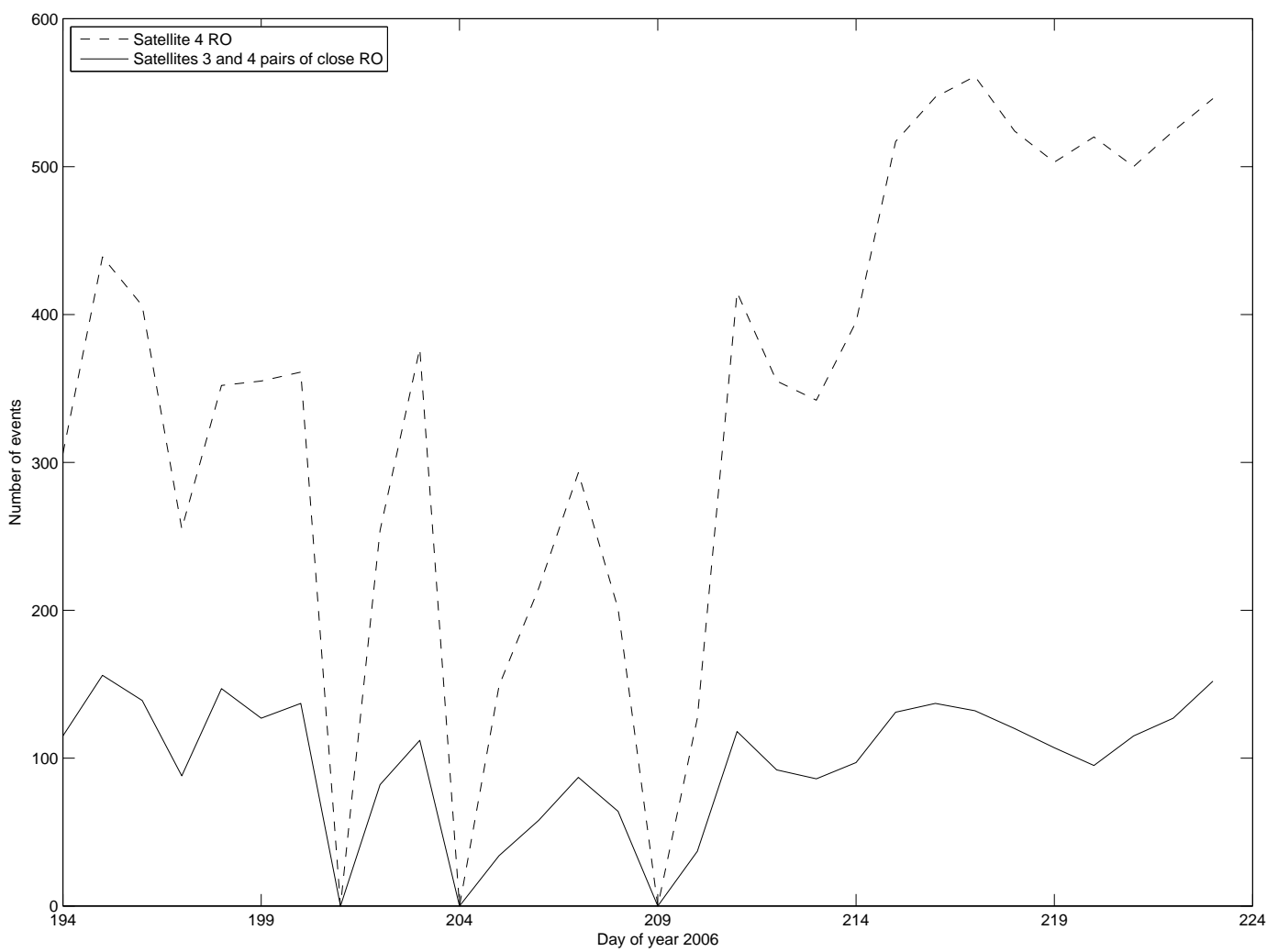

Figure 2: Number of RO retrievals for COSMIC satellite 4 and the number of pairs found with satellite 3 (both per day of year 2006). 

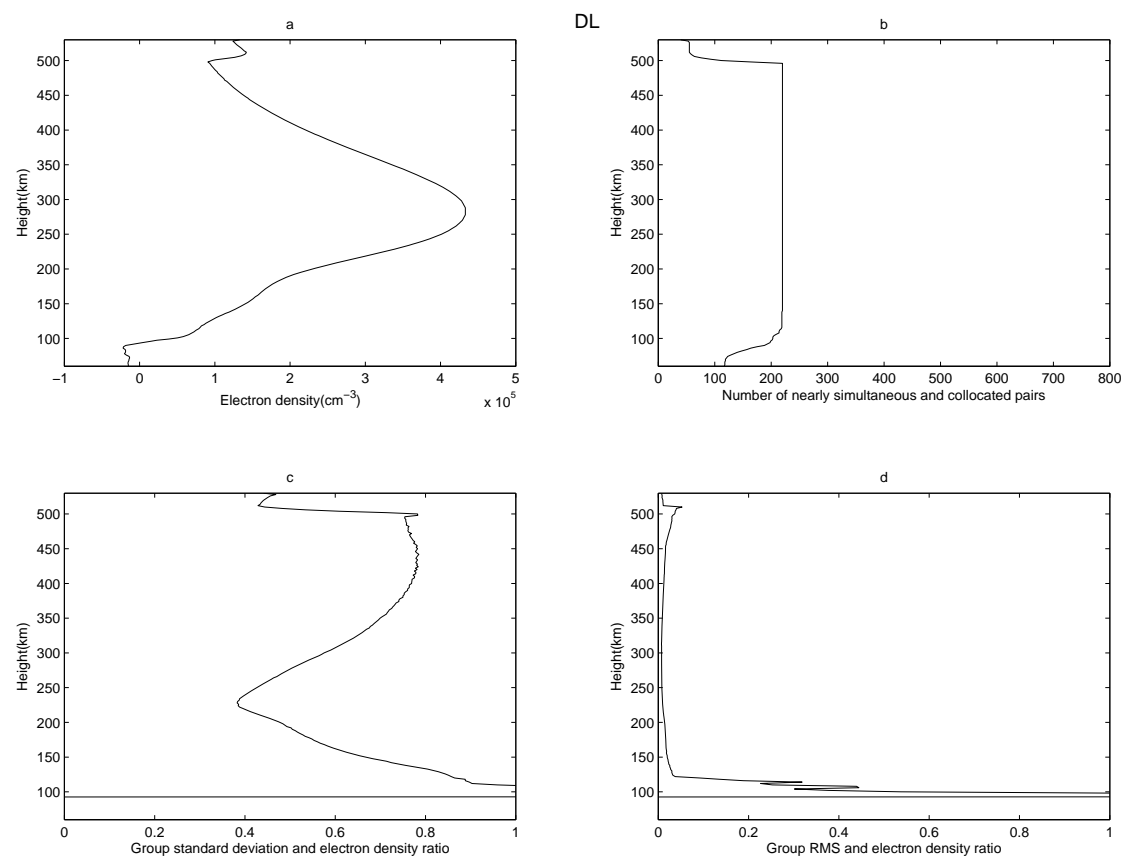

Figure 3: GPS RO profiles for daytime group DL against height of a) mean electron density, b) the number of pairs, c) the ratio of the standard deviation and the mean electron density, d) the ratio of the RMS of the pairs and the mean electron density. 

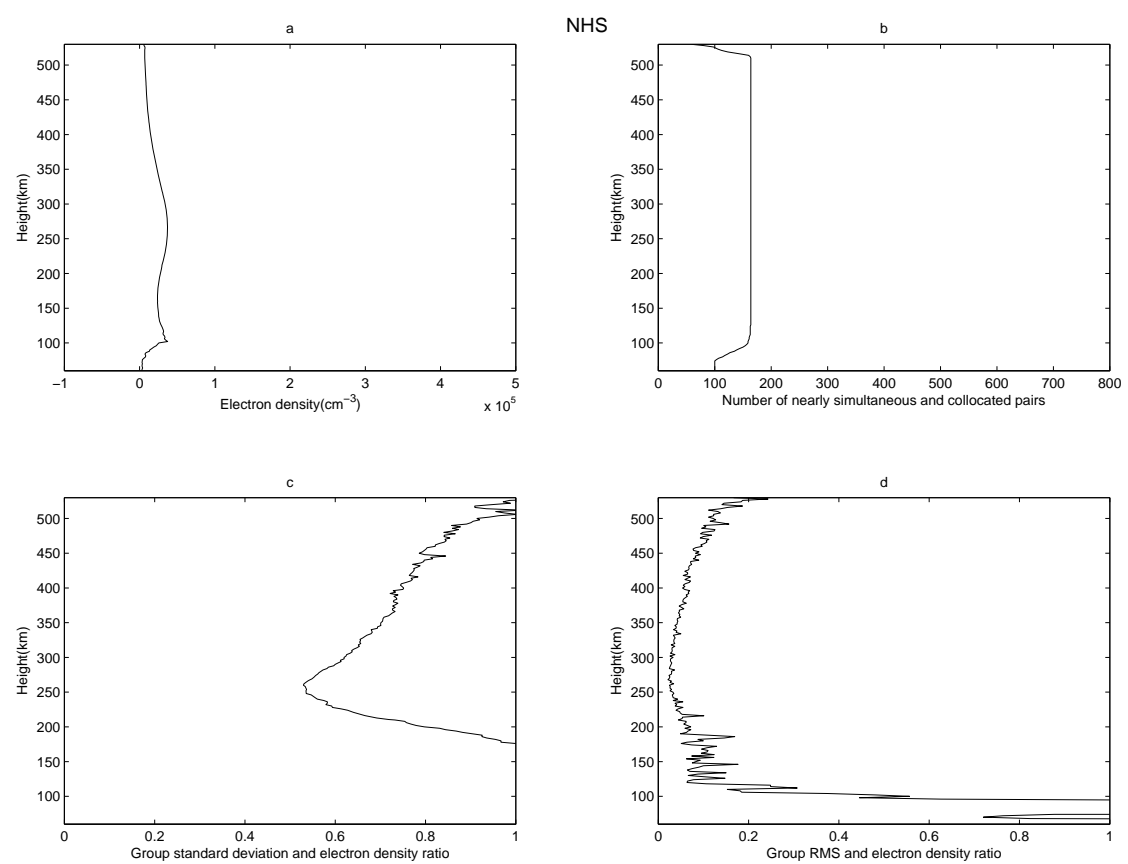

Figure 4: The same as Figure 3 for nighttime group NHS. 

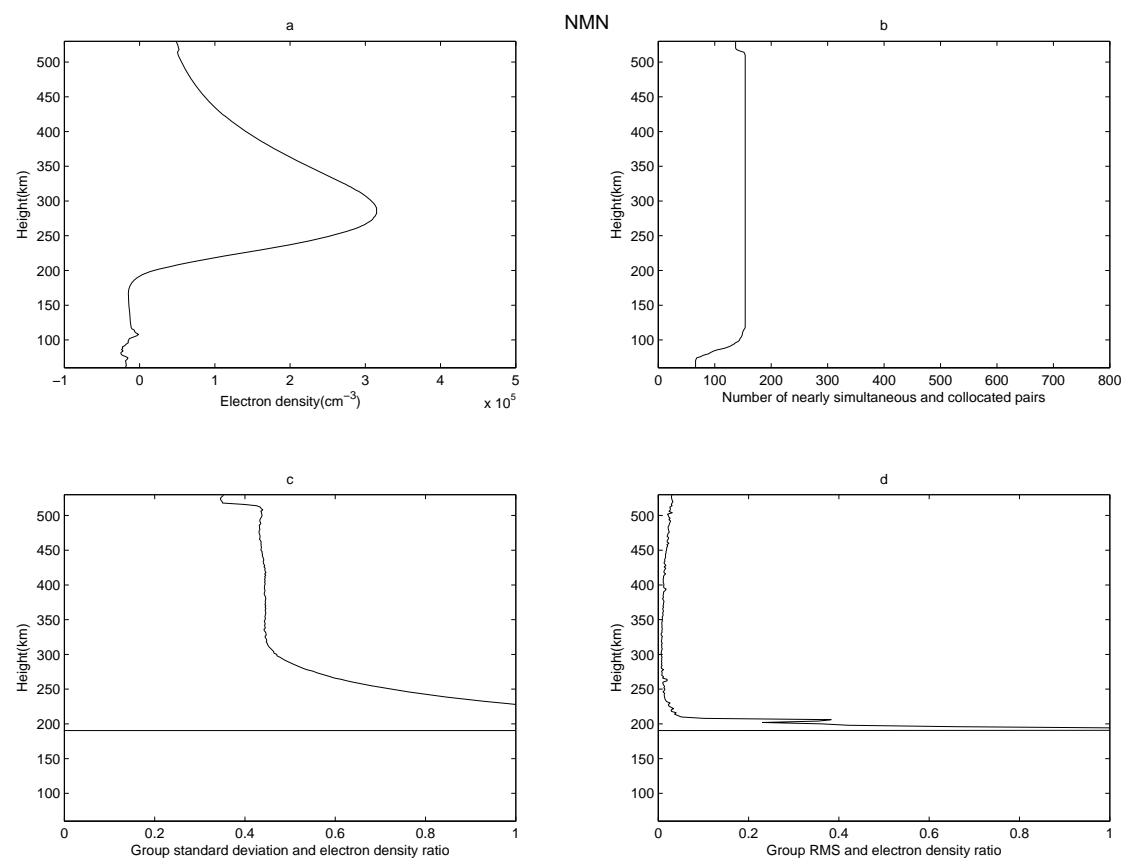

Figure 5: The same as Figure 3 for nighttime group NMN. 

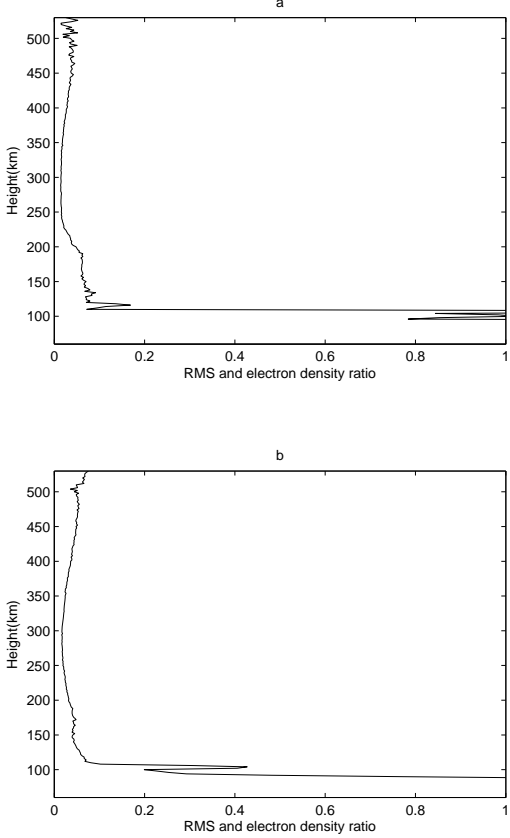

Figure 6: The ratio of RMS and mean electron density against height for the pairs which have an angle difference a) between $10^{\circ}$ and $20^{\circ}$, b) between $20^{\circ}$ and $40^{\circ}$. 

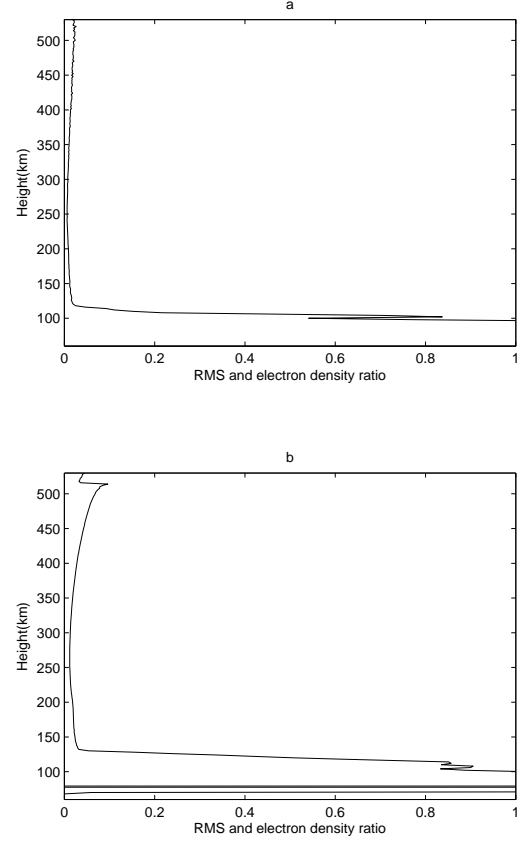

Figure 7: The ratio of RMS and mean electron density against height for a) the setting pairs of group DMN, b) the same for rising pairs. 


\section{Appendix A. The Abel transform}

In physical systems it is usual to encounter pairs of functions which are related by an integral relationship such as $T E C^{\prime}$ and $N_{e}$

$$
T E C^{\prime}\left(r_{o}\right) \approx 2 \int_{r_{o}}^{r_{L E O}} \frac{r N_{e}(r)}{\sqrt{r^{2}-r_{o}^{2}}} d r
$$

In a more general way it can be presented in the form

$$
f\left(r_{o}\right)=\int_{a}^{b} g(r) K\left(r_{o}, r\right) d r
$$

where the function $f\left(r_{o}\right)$ is called the integral transform of the function

$g(r)$ by the kernel $K\left(r_{o}, r\right)$. It cannot be expected that in general an inverse transform exists, but in some cases it can be found that

$$
g(r)=\int_{c}^{d} f\left(r_{o}\right) K^{-1}\left(r_{o}, r\right) d r_{o}
$$

The Abel transform can be defined as

$$
f\left(r_{o}\right)=\int_{0}^{\infty} g(r) K\left(r_{o}, r\right) d r
$$

where

$$
K\left(r_{o}, r\right)= \begin{cases}0 & \text { if } r \leq r_{o} \\ \frac{2 r}{\sqrt{r^{2}-r_{o}^{2}}} & \text { if } r>r_{o}\end{cases}
$$

The inverse kernel exists in this case

$$
K^{-1}\left(r_{o}, r\right)=-\frac{1}{\pi} \frac{1}{\sqrt{r_{o}^{2}-r^{2}}} \frac{d}{d r_{o}}
$$

so if the function $f\left(r_{o}\right)$ is differentiable

$$
g(r)=-\frac{1}{\pi} \int_{r}^{\infty} \frac{d f\left(r_{o}\right) / d r_{o}}{\sqrt{r_{o}^{2}-r^{2}}} d r_{o}
$$

